\title{
ESTUDO DA TERAPIA FOTODINÂMICA ASSOCIADA A NANOSSONDAS BIFUNCIONAIS PARA O TRATAMENTO DA LINHAGEM CELULAR MDA-MB-468
}

\author{
Mariana Lima Castilho' \\ Marcela Aparecida Cândido² \\ Bianca Silva Prado 3 \\ Lucas Pereira Leite ${ }^{4}$ \\ Kevin Cecil Hewitt ${ }^{5}$ \\ Leandro José Raniero ${ }^{6}$
}

Resumo: A nanotecnologia associada a diagnóstico e tratamento de câncer está emergindo como um novo campo de pesquisa interdisciplinar, focada na concepção e aplicação de nanomateriais. O direcionamento de ativos funcionalizados a nanopartículas para a terapia fotodinâmica (TFD) vem sendo estudado por ser menos agressivo e invasivo ao organismo. Neste âmbito, este trabalho propõe o desenvolvimento de uma nanopartícula de ouro bifuncional, composta de Clorina e6 (Ce6) com o direcionamento ativo por meio do fator de crescimento epidérmico, o qual foi testado na linhagem celular MDA-MB-468, via TFD. Os resultados mostraram uma viabilidade celular e atividade mitocondrial dez vezes menores em comparação com Ce6 livre, considerando a mesma concentração. A espectroscopia UV-Visível e o espalhamento dinâmico de luz foram utilizada na síntese e funcionalização das nanossondas bifuncionais.

Palavras-chave: Nanotecnologia; Terapia fotodinâmica; Câncer de mama; Clorina e6; Fator de Crescimento Epidérmico.

\footnotetext{
${ }^{1}$ Engenharia Biomédica/Universidade do Vale do Paraíba, Brasil. E-mail: mcastilho@univap.br.

2 Engenharia Química/Universidade do Vale do Paraíba, Brasil. E-mail: marcela.aparecida.candido@gmail.com.

${ }^{3}$ Biomedicina/Universidade do Vale do Paraíba, Brasil. E-mail: biancasilvaprado@hotmail.com.

${ }^{4}$ Biomedicina/Universidade do Vale do Paraíba, Brasil. E-mail: Iucaslleitepereira@gmail.com.

${ }^{5}$ Física/Dalhousie University, Canadá. E-mail: kevin.hewitt@dal.ca.

${ }^{6}$ Física/Universidade do Vale do Paraíba, Brasil. E-mail: Iraniero@univap.br.
} 